\title{
Relaxation-based color magnetic particle imaging for viscosity mapping
}

\author{
Cite as: Appl. Phys. Lett. 115, 152403 (2019); doi: 10.1063/1.5110475 \\ Submitted: 17 May 2019 - Accepted: 21 September 2019 . \\ Published Online: 9 October 2019
}

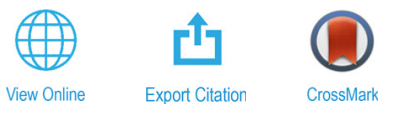

M. Utkur, ${ }^{1,2, a)}$ (D) Y. Muslu, ${ }^{1,2,3,4}$ (D) and E. U. Saritas ${ }^{1,2,5}$ (D)

\begin{abstract}
AFFILIATIONS
'Department of Electrical and Electronics Engineering, Bilkent University, Ankara 06800, Turkey

${ }^{2}$ National Magnetic Resonance Research Center (UMRAM), Bilkent University, Ankara 06800, Turkey

${ }^{3}$ Department of Biomedical Engineering, University of Wisconsin-Madison, Madison, Wisconsin 53706, USA

${ }^{4}$ Department of Radiology, University of Wisconsin-Madison, Madison, Wisconsin 53706, USA

${ }^{5}$ Neuroscience Program, Sabuncu Brain Research Center, Bilkent University, Ankara 06800, Turkey
\end{abstract}

${ }^{a)}$ Author to whom correspondence should be addressed: mustafa.utkur@bilkent.edu.tr

\begin{abstract}
Magnetic particle imaging (MPI) uses superparamagnetic iron oxide (SPIO) nanoparticles as biomedical imaging tracers. The potential applications of MPI have recently been broadened by the introduction of "color" MPI techniques that can distinguish different nanoparticles and/or environments, e.g., by exploiting the relaxation behavior of SPIOs. One of the important applications of color MPI techniques is viscosity mapping. In this work, we show relaxation-based color MPI experiments that can distinguish the biologically relevant viscosity range of up to $5 \mathrm{mPa}$. To find the optimal drive field parameters for viscosity, we compare color MPI results at three different frequencies. We show that frequencies around $10 \mathrm{kHz}$ are well-suited for viscosity mapping using the multicore cluster Nanomag-MIP nanoparticles, providing a one-to-one mapping between the estimated relaxation time constant and viscosity.
\end{abstract}

Published under license by AIP Publishing. https://doi.org/10.1063/1.5110475

Magnetic particle imaging (MPI) is a rapidly developing imaging modality that utilizes superparamagnetic iron oxide (SPIO) nanoparticles as tracers. ${ }^{1,2}$ The spatial distribution of the nanoparticles is mapped by exploiting their nonlinear magnetization response under an oscillating magnetic field. With MPI, highsensitivity and high-resolution images of the SPIO biodistribution can be obtained without any signal from the background tissue.

SPIOs exhibit relaxation, which delays the alignment of their internal magnetization with the applied magnetic field. Recently, a few groups have focused on understanding and utilizing this behavior for color MPI, with the purpose of differentiating different nanoparticle types and/or chemical environments. ${ }^{3-5}$ Color MPI techniques broaden MPI's capabilities to applications such as SPIO-labeled catheter tracking for vascular interventions, ${ }^{6}$ or steering of the catheters during endoscopic procedures. ${ }^{7,8}$ Color MPI studies can also be extended for functional imaging purposes to determine the binding state of the SPIOs, ${ }^{9}$ or to understand signaling and transportation in tissues. $^{10-12}$

One of the important application areas of color MPI is viscosity mapping. Certain diseases, such as atherosclerosis ${ }^{13}$ and cancer, ${ }^{14,15}$ are known to result in increased levels of cellular viscosity. These diseases can potentially be probed with MPI through measuring viscosity of the tissue where the SPIOs are located in. There are several methods in the MPI literature aimed at measuring viscosity, ${ }^{9,16-18}$ including our recently proposed technique, ${ }^{19}$ and it is essential to incorporate these methods into in vivo imaging applications.

In this study, we demonstrate imaging results of relaxationbased color MPI for viscosity mapping. We base the color MPI experiments on our recently proposed relaxation time constant estimation technique, ${ }^{5,19}$ briefly referred to as time constant $(\tau$, TAU) estimation via Recovery of Underlying mirror Symmetry (TAURUS). Comparing the results from our in-house MPI scanner tuned to three different drive field (DF) frequencies, we show that frequencies around $10 \mathrm{kHz}$ provide one-to-one mapping between the estimated time constant and viscosity for multicore cluster Nanomag-MIP nanoparticles. We present 2D color MPI results that differentiate different viscosity levels in the biologically relevant range of up to $5 \mathrm{mPa}$.

In MPI, the relaxation behavior of SPIOs causes the signal to be delayed in time and reduced in amplitude. In X-space MPI, this relaxation effect was modeled via an exponential kernel with time constant $\tau$, using the following formalism: ${ }^{2}$ 


$$
s(t)=s_{\text {ideal }}(t) *\left\{\frac{1}{\tau} e^{\frac{-t}{\tau}} \mathcal{u}(t)\right\} .
$$

Here, $u(t)$ is the Heaviside step function and “*” denotes convolution. For a periodic DF, the ideal signal, $s_{\text {ideal }}(t)$, has symmetric negative and positive half cycles. However, the actual MPI signal, $s(t)$, loses this symmetry property due to relaxation induced blurring along the scanning direction. We have previously shown with our TAURUS technique that $\tau$ can be directly estimated from $s(t)$ as follows: ${ }^{5,19}$

$$
\tau=\frac{S_{\text {pos }}^{*}(f)+S_{\text {neg }}(f)}{i 2 \pi f\left(S_{\text {pos }}^{*}(f)-S_{\text {neg }}(f)\right)} .
$$

Here, $S_{\text {pos }}(f)$ and $S_{\text {neg }}(f)$ are Fourier transforms of the positive and negative half cycles of $s(t)$, respectively. The $*$ symbol in the superscript denotes the conjugation operation. When the signal is deconvolved with an exponential kernel using the correct $\tau$ value, the underlying mirror symmetry can be recovered. Importantly, the TAURUS technique does not require any prior information about the nanoparticles when estimating $\tau$.

An imaging phantom that contained nanoparticle samples at 5 different viscosity levels was prepared using varying ratios of water/ glycerol mixtures, as shown in Fig. 1(a). Each sample was prepared in a capillary tube with a $2 \mathrm{~mm}$ diameter, had a total volume of $20 \mu \mathrm{l}$, and contained $5 \mu \mathrm{l}$ of undiluted Nanomag-MIP nanoparticles ${ }^{38}$ (plain, $c(\mathrm{Fe})=89 \mathrm{mmol} \mathrm{Fe} / \mathrm{l}$, Micromod $\mathrm{GmbH}$, Germany). The resulting samples had viscosity levels ranging between $0.89 \mathrm{mPas}$ and 5.04 $\mathrm{mPa}$, covering biologically relevant viscosity levels. ${ }^{21,22}$ Table I lists the details of each nanoparticle sample.
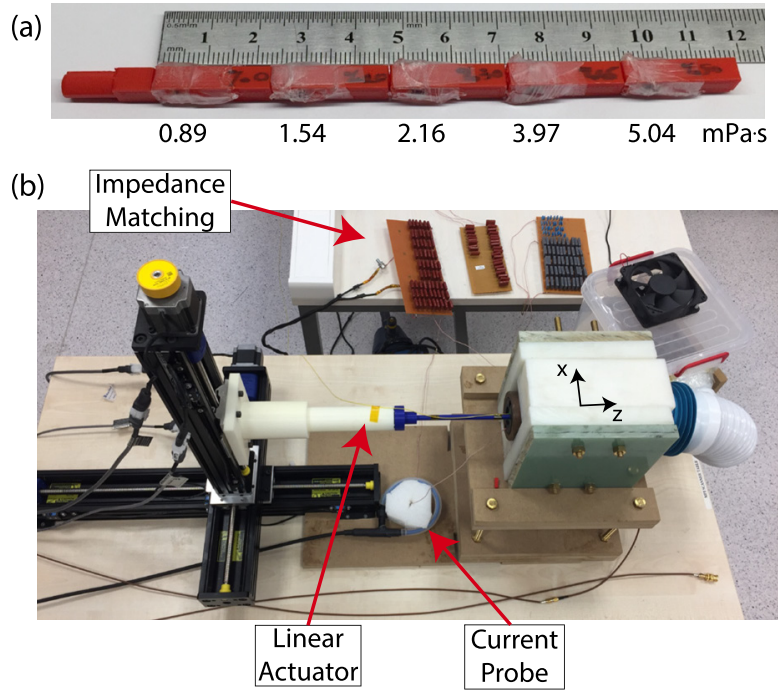

FIG. 1. (a) The imaging phantom for color MPI experiments. Nanoparticle samples at 5 different viscosity levels ranging between $0.89 \mathrm{mPas}$ and $5.04 \mathrm{mPas}$ were placed in a 3-D printed custom sample holder, where the placements of the samples can be interchanged. (b) Our in-house MPI scanner with $(-4.8,2.4,2.4)$ $\mathrm{T} / \mathrm{m} / \mu_{0}$ gradients in $(x, y, z)$ directions. The drive coil was impedance matched at three different frequencies: $1.1 \mathrm{kHz}, 9.7 \mathrm{kHz}$, and $26.3 \mathrm{kHz}$. The imaging phantom was moved in the $x-z$ plane using a linear actuator. A current probe was used to calibrate the drive field amplitude.
TABLE I. The contents and viscosity levels $(\eta)$ at $25^{\circ} \mathrm{C}$ for the nanoparticle samples. Each sample started from $5 \mu \mathrm{l}$ of undiluted Nanomag-MIP nanoparticles suspended in water and had a final volume of $20 \mu \mathrm{l}$. The table lists the added glycerol and water volumes and the final glycerol percentage by volume.

\begin{tabular}{ccccc}
\hline \hline$\#$ & $\eta(\mathrm{mPas})$ & Glycerol $(\mu \mathrm{l})$ & Water $(\mu \mathrm{l})$ & Glycerol \% \\
\hline 1 & 0.89 & 0 & 15 & 0 \\
2 & 1.54 & 4 & 11 & 20 \\
3 & 2.16 & 6 & 9 & 30 \\
4 & 3.97 & 9 & 6 & 45 \\
5 & 5.04 & 10 & 5 & 50 \\
\hline \hline
\end{tabular}

The imaging experiments were performed on our in-house MPI scanner. ${ }^{5,23}$ The selection field was generated using permanent disc-shaped magnets, resulting in $(-4.8,2.4,2.4) \mathrm{T} / \mathrm{m} / \mu_{0}$ gradients in $(\mathrm{x}, \mathrm{y}, \mathrm{z})$ directions [see Fig. 1(b)]. Using capacitive L-section circuits, the drive coil was impedance matched to the power amplifier (AE Techron 7224) at three different frequencies: $1.1 \mathrm{kHz}, 9.7 \mathrm{kHz}$, and $26.3 \mathrm{kHz}$. The drive coil was air-cooled to prevent heating of the scanner bore and nanoparticle samples. The scanner bore had an axial diameter of $1 \mathrm{~cm}$ in the $\mathrm{x}-\mathrm{y}$ plane, with a flexible length along the $\mathrm{z}$-direction. The drive field was applied along the $\mathrm{z}$-direction with amplitudes varying between $10 \mathrm{mT}$ and $25 \mathrm{mT}$, corresponding to partial field-of-view (pFOV) sizes between $4.2 \mathrm{~mm}$ and $10.4 \mathrm{~mm}$. The DF amplitude was calibrated with a current probe (LFR 06/6/300) promptly before each measurement. For all the $1 \mathrm{D}$ and $2 \mathrm{D}$ imaging experiments, the phantom was mechanically moved to the center of each pFOV using a linear actuator (Velmex BiSlide), with $85 \%$ overlap between neighboring pFOVs. For each pFOV, the signal was filtered and processed as previously described. ${ }^{5} \mathrm{MPI}$ images were then reconstructed using a signal-to-noise ratio (SNR) optimized $\mathrm{x}$-space reconstruction $^{24}$ with the DC recovery algorithm. ${ }^{25}$ The relaxation time constant maps ( $\tau$ maps) were directly estimated from the timedomain signals for each pFOV, using the aforementioned TAURUS technique.

First, six different 1D imaging experiments (at 3 different DF frequencies and 2 different DF amplitudes) were conducted to determine the optimal DF parameters for relaxation-based viscosity mapping. The DF amplitudes tested were $10 \mathrm{mT}$ and $15 \mathrm{mT}$ at $1.1 \mathrm{kHz}$ and $9.7 \mathrm{kHz}$ and $10 \mathrm{mT}$ and $14 \mathrm{mT}$ at $26.3 \mathrm{kHz}$ (the latter restricted by hardware limitations). Experiments were first performed with the samples ordered randomly (results not shown) and then with the samples ordered in increasing viscosity levels. Both cases yielded matching results, ensuring that there was no position-induced bias. The resulting $1 \mathrm{D}$ MPI images and $\tau$ maps were replicated along the vertical direction for display purposes, as shown in Figs. 2(a) and 2(b). Note that for simplicity of labeling, the results at $26.3 \mathrm{kHz}$ and $14 \mathrm{mT}$ are grouped under $15 \mathrm{mT}$ results.

As seen in Fig. 2(b), $\tau$ maps at $26.3 \mathrm{kHz}$ appear flat. Hence, although frequencies around $25 \mathrm{kHz}$ are widely popular in MPI, this high frequency range is not favorable for probing viscosity. On the other hand, $\tau$ maps at $1.1 \mathrm{kHz}$ and $9.7 \mathrm{kHz}$ display a variety of colors, indicating a viscosity-sensitive contrast capability. Furthermore, the MPI images at these lower frequencies display better resolution, which is consistent with the literature. ${ }^{26,27}$ Next, mean $\tau$ values were 


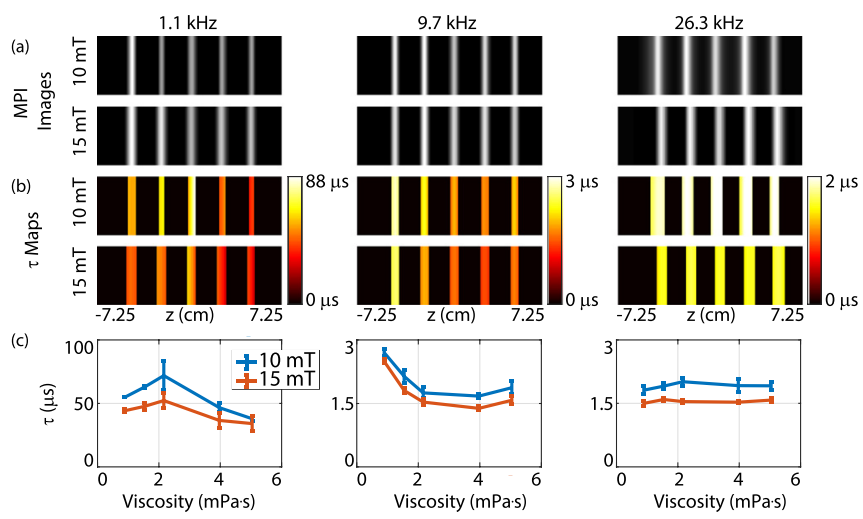

FIG. 2. 1D color MPI results at 3 different drive field frequencies and 2 different drive field amplitudes, for the phantom shown in Fig. 1(a) where the samples were ordered in increasing viscosity levels between $0.89 \mathrm{mPas}$ and $5.04 \mathrm{mPas}$. (a) 1D MPI images and (b) $1 \mathrm{D}$ relaxation $(\tau)$ maps, replicated in the vertical direction for display purposes. (c) $\tau$ vs viscosity curves, extracted from the $\tau$ maps. The predominantly monotonic trend at $9.7 \mathrm{kHz}$ indicates that this drive field frequency is well-suited for one-to-one viscosity mapping. The error bars denote the mean values and standard deviations computed from corresponding ROls in $\tau$ maps for each sample.

computed from the corresponding regions-of-interest (ROIs) in $\tau$ maps and plotted as a function of viscosity in Fig. 2(c). Accordingly, there is a considerable differentiation in $\tau$ values with respect to viscosity at $9.7 \mathrm{kHz}$. At $1.1 \mathrm{kHz}$ and $26.3 \mathrm{kHz}$, on the other hand, the curves are either nonmonotonic or remain approximately flat.

The trends observed in Fig. 2 are consistent with our previous experimental work in a magnetic particle spectrometer (MPS) setup, ${ }^{19}$ with one important difference: In the MPS setup, $\tau$ vs viscosity curves displayed a nonmonotonic trend at lower DF frequencies of up to $550 \mathrm{~Hz}$, switched to a monotonic trend at $1.1 \mathrm{kHz}$, and finally became flat at $10.8 \mathrm{kHz}$. In this work, with the added selection field of an MPI scanner, the switch from the nonmonotonic to monotonic trend occurs at a higher frequency range. A side-by-side comparison of normalized $\tau$ values from MPS and MPI experiments is shown in Fig. 3,
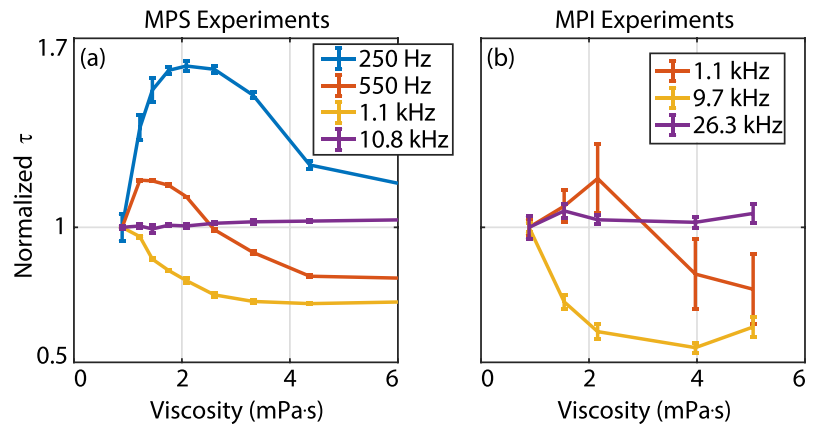

FIG. 3. Comparison of $\tau$ vs viscosity curves for (a) MPS experiments (replotted from Ref. 19) and (b) color MPI experiments, performed at different drive field frequencies at a $15 \mathrm{mT}$ amplitude. The results are color matched to highlight similar trends. A monotonic $\tau$ vs viscosity curve is observed at $1.1 \mathrm{kHz}$ in MPS experiments, whereas a similar trend is achieved at $9.7 \mathrm{kHz}$ with the additional selection field of the MPI scanner. color matched to highlight similar trends at different frequencies. The trend seen at $550 \mathrm{~Hz}$ in the MPS experiments is similar to that at $1.1 \mathrm{kHz}$ in the MPI experiments. Likewise, $1.1 \mathrm{kHz}$ and $10.8 \mathrm{kHz}$ in MPS experiments display similar trends to $9.7 \mathrm{kHz}$ and $26.3 \mathrm{kHz}$ in MPI experiments, respectively. Importantly, for MPI experiments, the monotonic $\tau$ vs viscosity curve at $9.7 \mathrm{kHz}$ indicates that this operating frequency is highly promising for one-to-one mapping of viscosity from a measured $\tau$ value.

To further investigate the viscosity mapping capability at $9.7 \mathrm{kHz}$, $1 \mathrm{D}$ imaging experiments were performed at four different DF amplitudes between $10 \mathrm{mT}$ and $25 \mathrm{mT}$. The corresponding MPI images, $\tau$ maps, and $\tau$ vs viscosity curves are displayed in Fig. 4 . At this frequency, $\tau$ values vary by more than $50 \%$ in the tested viscosity range, which is a highly desirable feature for mapping purposes. Note that a superior monotonicity is reached at a $25 \mathrm{mT} \mathrm{DF}$ amplitude. Furthermore, given that the MPI signal increases linearly with the DF amplitude, ${ }^{27}$ the increased SNR at $25 \mathrm{mT}$ results in reduced deviation in $\tau$ estimations [see error bars in Fig. 4(c)].

Finally, a 2D color MPI demonstration was performed at the optimal DF parameters: $9.7 \mathrm{kHz}$ and $25 \mathrm{mT}$. The phantom in Fig. 5(a) was scanned with a FOV of $0.8 \mathrm{~cm} \times 14.5 \mathrm{~cm}$ in $\mathrm{x}$ - and z-directions, respectively. This FOV was rastered along the z-direction as 9 evenly spaced lines, each made up of 39 overlapping pFOVs. The resulting 2D MPI image and $\tau$ map, as well as their color overlay, are shown in Figs. 5(b)-5(d). The nanoparticle samples at different viscosity levels are easily distinguishable in the $\tau$ map, confirming the suitability of these DF parameters for one-to-one viscosity mapping.

In MPI, the properties of the SPIOs such as their size, dispersity, and anisotropy have a large impact on their signal. ${ }^{28}$ These factors affect the performance of the SPIOs not just in MPI but also in other modalities such as magnetic resonance imaging (MRI) or magnetic fluid hyperthermia (MFH). ${ }^{29,30}$ Similarly, the optimal DF parameters may depend on the nanoparticle type. In a previous work, we compared $\tau$ vs viscosity trends for two multicore SPIOs, Nanomag-MIP and VivoTrax, where the latter has the same chemical structure as Resovist. We observed that VivoTrax displayed similar global trends to Nanomag-MIP, but at higher DF frequencies. ${ }^{19}$ Therefore, the optimal DF parameters, particularly the DF frequency, may need to be tuned for a given SPIO type.

Brownian and Néel relaxation time constants of SPIOs are normally calculated using zero-field formulas, which are not valid for the

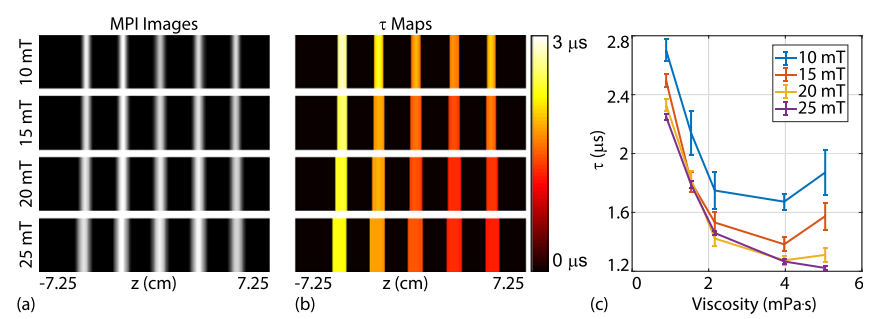

FIG. 4. 1D color MPI results at 4 different drive field amplitudes at $9.7 \mathrm{kHz}$, for the phantom in Fig. 5(a) where samples are ordered in increasing viscosity levels. (a) 1D MPI images and (b) $\tau$ maps, replicated in the vertical direction for display purposes. (c) $\tau$ vs viscosity curves indicate that a higher drive field amplitude of $25 \mathrm{mT}$ yields superior monotonicity. The error bars denote the mean values and standard deviations computed from the corresponding ROls in $\tau$ maps for each sample. 


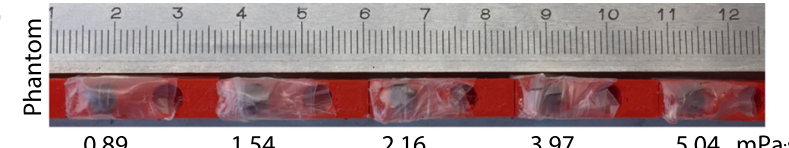

(b)

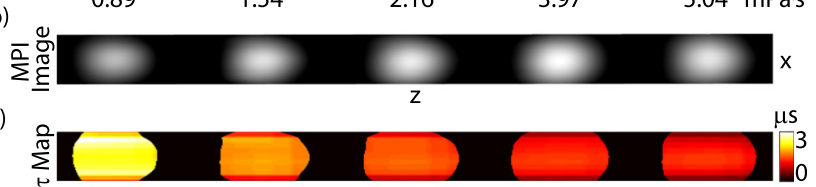

(d)

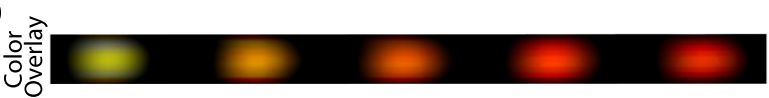

FIG. 5. 2D color MPI demonstration at the optimal drive field parameters: $9.7 \mathrm{kHz}$ and $25 \mathrm{mT}$. (a) The imaging phantom with 5 different samples ordered in increasing viscosity levels ranging between $0.89 \mathrm{mPa}$ and $5.04 \mathrm{mPa}$. (b) 2D MPI image, (c) 2D $\tau$ map, and (d) their color overlay. Different viscosity levels are easily distinguishable in the $\tau$ map, as well as the color overlay. The phantom was scanned with a FOV of $0.8 \mathrm{~cm} \times 14.5 \mathrm{~cm}$ in $\mathrm{x}$ - and z-directions, respectively. The displayed FOV is $0.8 \mathrm{~cm} \times 11.5 \mathrm{~cm}$

sinusoidal DF in MPI. ${ }^{31}$ In $\tau$ vs viscosity curves at lower DF frequencies/amplitudes, the estimated $\tau$ values initially increase with increasing viscosity, ${ }^{19}$ seemingly obeying Brownian relaxation dynamics. At higher frequencies/amplitudes/viscosities, however, this initial trend can no longer be observed. We believe that the reason behind this phenomenon is that the nanoparticle magnetization does not have sufficient time to reach its steady-state value under these "more demanding" cases. Hence, one can no longer observe the expected monotonic increase with increasing viscosity, causing a different trend to emerge. A similar phenomenon was also described in a recent work, where it was shown that the magnetization could not reach its steadystate value (i.e., the value dictated by Langevin physics) in the case of square-wave pulsed excitations with higher frequencies/amplitudes. ${ }^{32}$

The above-cited work has also shown that nanoparticles farther from the field free point (FFP) are characterized with faster relaxation when compared to the nanoparticles closer to the FFP. ${ }^{32}$ Likewise, preliminary experiments on our MPS setup also demonstrated that $\tau$ values can be reduced in the presence of an additional DC field (results not shown). Importantly, the change in $\tau$ values can be at different rates for different viscosity levels, which in turn can change the overall $\tau$ vs viscosity trend. Hence, under the static selection field of an MPI scanner, the observed $\tau$ values as well as the $\tau$ vs viscosity trend can change when compared to those in an MPS setup. Understanding the physical reasons behind the latter effect requires future experiments and analysis.

Previous work has shown that SNR scales almost linearly with the product of the DF frequency and amplitude. ${ }^{27}$ Therefore, even if a monotonic trend could be achieved at a lower DF frequency/amplitude than the tested range, the SNR of the images/maps would suffer considerably. For example, $1.1 \mathrm{kHz}$ and $5 \mathrm{mT}$ would yield approximately 44 times lower SNR than at $9.7 \mathrm{kHz}$ and $25 \mathrm{mT}$, which is a massive difference. At very high frequencies/amplitudes, on the other hand, the $\tau$ vs viscosity trends flatten out. ${ }^{19}$ Hence, our goal in this work was to operate at as high a frequency/amplitude as possible, while attaining a one-to-one mapping between $\tau$ and viscosity in the biologically relevant range.

Even when operating at high DF frequency/amplitude, SNR can be low in in vivo settings. A noise robustness of the TAURUS technique was previously performed, ${ }^{5}$ showing that even at a very low SNR value of 2 , the relaxation time constant can be estimated with a standard deviation that is less than $0.4 \%$ of the drive field period (around $0.4 \mu \mathrm{s}$ at $9.7 \mathrm{kHz}$ ). For SNR values greater than 15, the standard deviations rapidly decrease to less than $0.05 \%$ of the drive field period (around $0.05 \mu$ s at $9.7 \mathrm{kHz}$ ). The specific iron amount corresponding to a given SNR level depends on the scanner geometry and nanoparticle type, as well as the scanning parameters. ${ }^{33}$ Considering that MPI features a state-of-the-art detection limit on the order of 5-100 $\mathrm{ng} \mathrm{Fe},{ }^{34-36}$ with a theoretical picogram sensitivity capability, ${ }^{33,37}$ the SNR robustness of the TAURUS technique makes it an excellent candidate for in vivo viscosity mapping with MPI.

Here, we have demonstrated the TAURUS technique using multicore cluster nanoparticles. In in vivo settings, size selective uptake may take place during the internalization of the nanoparticles into the tissue/cells, which may change the MPI response for nanoparticles that contain different sized cores. To avoid such effects, single-core nanoparticles may be preferred. Investigating such in vivo challenges and potential solutions for the proposed technique remains a future work.

To summarize, this work demonstrates the viscosity mapping capability of MPI for functional imaging applications. With our in-house MPI scanner, we showed that a one-to-one mapping between the relaxation time constant and viscosity can be achieved at drive field parameters around $10 \mathrm{kHz}$ and $25 \mathrm{mT}$ using multicore cluster Nanomag-MIP nanoparticles. These results present relaxation-based color MPI experiments distinguishing the biologically relevant viscosity range.

The authors thank Ugur Yilmaz for the valuable discussions. This work was supported by the Scientific and Technological Research Council of Turkey (No. TUBITAK 115E677).

\section{REFERENCES}

1B. Gleich and J. Weizenecker, Nature 435, 1214-1217 (2005).

${ }^{2}$ J. Weizenecker, J. Borgert, and B. Gleich, Phys. Med. Biol. 52, 6363-6374 (2007).

3J. Rahmer, A. Halkola, B. Gleich, I. Schmale, and J. Borgert, Phys. Med. Biol. 60, 1775-1791 (2015)

${ }^{4}$ D. Hensley, P. Goodwill, L. Croft, and S. Connoly, in Proceedings of the 5th International Workshop on Magnetic Particle Imaging (IWMPI), Istanbul (2015).

${ }^{5}$ Y. Muslu, M. Utkur, O. B. Demirel, and E. U. Saritas, IEEE TMI 37, 1920-1931 (2018).

${ }^{6}$ J. Haegele, N. Panagiotopoulos, S. Cremers, J. Rahmer, J. Franke, R. L. Duschka, S. Vaalma, M. Heidenreich, J. Borgert, P. Borm, J. Barkhausen, and F. M. Vogt, IEEE TMI 35, 2312-2318 (2016).

${ }^{7}$ N. Nothnagel, J. Rahmer, B. Gleich, A. Halkola, T. M. Buzug, and J. Borgert, IEEE Trans. Biomed. Eng. 63, 2286-2293 (2016).

${ }^{8}$ J. Rahmer, D. Wirtz, C. Bontus, J. Borgert, and B. Gleich, IEEE TMI 36, 1449-1456 (2017).

${ }^{9}$ A. M. Rauwerdink and J. B. Weaver, J. Magn. Magn. Mater. 322, 609-613 (2010).

${ }^{10}$ S. P. Williams, P. M. Haggie, and K. M. Brindle, Biophys. J. 72, 490-498 (1997).

${ }^{11}$ A. M. Rauwerdink and J. B. Weaver, Appl. Phys. Lett. 96, 033702 (2010).

${ }^{12}$ A. M. Rauwerdink and J. B. Weaver, Med. Phys. 38, 1136-1140 (2011).

${ }^{13}$ G. Deliconstantinos, V. Villiotou, and J. C. Stavrides, Biochem. Pharmacol. 49, 1589-1600 (1995).

${ }^{14}$ W. L. Chandler and G. Schmer, Clin. Chem. 32, 505-507 (1986).

${ }^{15} \mathrm{G}$. F. von Tempelhoff, N. Schonmann, L. Heilmann, K. Pollow, and G. Hommel, Clin. Hemorheol. Microcirc. 26, 55-61 (2002).

${ }^{16}$ J. B. Weaver and E. Kuehlert, Med. Phys. 39, 2765-2770 (2012). 
${ }^{17}$ T. Viereck, C. Kuhlmann, S. Draack, M. Schilling, and F. Ludwig, J. Magn. Magn. Mater. 427, 156-161 (2017).

${ }^{18}$ M. Möddel, C. Meins, J. Dieckhoff, and T. Knopp, New J. Phys. 20, 083001 (2018).

${ }^{19}$ M. Utkur, Y. Muslu, and E. U. Saritas, Phys. Med. Biol. 62, 3422-3439 (2017).

${ }^{20}$ L. R. Croft, P. W. Goodwill, and S. M. Conolly, IEEE TMI 31, 2335-2342 (2012).

${ }^{21}$ M. K. Kuimova, S. W. Botchway, A. W. Parker, M. Balaz, H. A. Collins, H. L. Anderson, K. Suhling, and P. R. Ogilby, Nat. Chem. 1, 69-73 (2009).

${ }^{22}$ B. Pirofsky, J. Clin. Invest. 32, 292-298 (1953).

${ }^{23}$ M. Utkur, Y. Muslu, and E. U. Saritas, in Proceedings of 21st National Biomedical Engineering Meeting (BIYOMUT), Istanbul (2017).

${ }^{24}$ E. Bozkurt and E. U. Saritas, J. Fac. Eng. Archit. Gazi Univ. 32, 999-1013 (2017).

${ }^{25} \mathrm{~K}$. Lu, P. W. Goodwill, E. U. Saritas, B. Zheng, and S. M. Conolly, IEEE TMI 32, 1565-1575 (2013).

${ }^{26}$ A. Weber, J. Weizenecker, J. Rahmer, J. Franke, U. Heinen, and T. Buzug, in Proceedings of the 5th International Workshop on Magnetic Particle Imaging (IWMPI), Istanbul (2015).

${ }^{27}$ L. R. Croft, P. W. Goodwill, J. Konkle, H. Arami, D. A. Price, A. X. Li, E. U. Saritas, and S. M. Conolly, Med. Phys. 43, 424-435 (2016).

${ }^{28}$ A. P. Khandhar, R. M. Ferguson, H. Arami, and K. M. Krishnan, Biomaterials 34, 3837-3845 (2013).
${ }^{29}$ A. Alipour, Z. Soran-Erdem, M. Utkur, V. K. Sharma, O. Algin, E. U. Saritas, and H. V. Demir, Magn. Reson. Imaging 49, 16-24 (2018).

${ }^{30}$ R. Dhavalikar, A. C. Bohorquez, and C. Rinaldi, Nanomaterials for Magnetic and Optical Hyperthermia Applications (Elsevier, Cambridge, MA, USA, 2019), pp. 265-286.

${ }^{31}$ R. J. Deissler and M. A. Martens, IEEE Trans. Magn. 51, 1-4 (2015).

${ }^{32}$ Z. W. Tay, D. Hensley, J. Ma, P. Chandrasekharan, B. Zheng, P. Goodwill, and S. Conolly, IEEE Trans. Med. Imaging 38, 2389-2399 (2019).

${ }^{33}$ X. Y. Zhou, K. E. Jeffris, E. Y. Yu, B. Zheng, P. W. Goodwill, P. Nahid, and S. M. Conolly, Phys. Med. Biol. 62, 3510-3522 (2017).

${ }^{34}$ B. Zheng, T. Vazin, P. W. Goodwill, A. Conway, A. Verma, E. U. Saritas, D. Schaffer, and S. M. Conolly, Sci. Rep. 5, 14055 (2015).

${ }^{35}$ B. Zheng, E. Yu, R. Orendorff, K. Lu, J. J. Konkle, Z. W. Tay, D. Hensley, X. Y. Zhou, P. Chandrasekharan, E. U. Saritas, P. W. Goodwill, J. D. Hazle, and S. M. Conolly, Mol. Imaging Biol. 19, 385-390 (2017).

${ }^{36}$ M. Graeser, T. Knopp, P. Szwargulski, T. Friedrich, A. von Gladiss, M. Kaul, K. M. Krishnan, H. Ittrich, G. Adam, and T. M. Buzug, Sci. Rep. 7, 6872 (2017).

${ }^{37}$ B. Gleich, Principles and Applications of Magnetic Particle Imaging (Springer Fachmedien Wiesbaden, 2014).

${ }^{38}$ D. Eberbeck, C. L. Dennis, N. F. Huls, K. L. Krycka, C. Gruttner, and F. Westphal, IEEE Trans. Magn. 49, 269-274 (2013). 